\title{
Development and characterization of a packaging cell line for pseudo-infectious yellow fever virus particle generation
}

\author{
Sabrina Ribeiro de Almeida Queiroz ${ }^{[1]}$, José Valter Joaquim Silva Júnior ${ }^{[1]}$, \\ Andréa Nazaré Monteiro Rangel da Silva ${ }^{[2]}$, Amanda Gomes de Oliveira Carvalho ${ }^{[1],}$ \\ Jefferson José da Silva Santos ${ }^{[3]}$ and Laura Helena Vega Gonzales Gil ${ }^{[1]}$
}

\begin{abstract}
[1].Departamento de Virologia e Terapia Experimental, Instituto Aggeu Magalhães, Fundação Oswaldo Cruz, Recife, PE, Brasil. [2]. Laboratório de Virologia, Instituto de Ciências Biológicas, Universidade Federal do Pará-UFPA, Belém, Pará, Brasil. [3]. Department of Population Health, Poultry Diagnostic and Research Center, University of Georgia, Athens, USA.
\end{abstract}

\begin{abstract}
Introduction: Pseudo-infectious yellow fever viral particles (YFV-PIVs) have been used to study vaccines and viral packaging. Here, we report the development of a packaging cell line, which expresses the YFV prM/E proteins. Methods: HEK293 cells were transfected with YFV prM/E and C (84 nt) genes to generate HEK293-YFV-PrM/E-opt. The cells were evaluated for their ability to express the heterologous proteins and to package the replicon repYFV-17D-LucIRES, generating YFV-PIVs. Results: The expression of $\mathrm{prM} / \mathrm{E}$ proteins was confirmed, and the cell line trans-packaged the replicon for recovery of a reporter for the YFV-PIVs. Conclusions: HEK293-YFV-prM/E-opt trans-packaging capacity demonstrates its possible biotechnology application.
\end{abstract}

Keywords: Yellow fever virus. Trans-packaging. Pseudo-infectious particles.

The yellow fever virus (YFV) (genus Flavivirus, family Flaviviridae) consists of a positive-sense, single-stranded RNA genome, approximately $11 \mathrm{~kb}$ in size, with a single open reading frame (ORF), flanked by two untranslated regions (UTR): one at the 5' end (with a Cap structure) and the other at the 3' end (without a polyadenylated tail). The ORF encodes a single polyprotein that is processed by viral and host proteases to generate three structural proteins (C-prM/M-E), with morphogenic and adsorption functions, and seven nonstructural proteins (NS1-NS2A-NS2B-NS3-NS4A-NS4B-NS5) responsible for viral replication and evasion of the immune response ${ }^{1}$.

Yellow fever is an arboviral disease with a clinical spectrum ranging from asymptomatic to severe. In severe cases, disease progression is marked by intense muscle weakness, nausea, vomiting, jaundice, headaches, lower back pain, renal failure, shock, and hemorrhage, which often manifests as Faget's sign. Death occurs in 20 to $50 \%$ of severe cases and is commonly preceded by deep jaundice, hemorrhage, hypotension, oliguria, and azotemia ${ }^{2}$.

Corresponding author: Dra. Laura Helena Vega Gonzales Gil.

e-mail: laura@cpqam.fiocruz.br

Received 1 June 2017

Accepted 12 September 2017
In Brazil, a recent urban outbreak was reported between December 2016 and May 4, 2017, with 729 confirmed cases of the disease and another 663 under investigation. During that time, 249 deaths were due to yellow fever, and 45 remain under investigation ${ }^{3}$.

The yellow fever vaccine was developed in 1936 by attenuating an African sample of the wild-type yellow fever virus called Asibi. Currently, two main live-attenuated licensed vaccines are available: one uses the 17D-204 strain, and the other, which has been produced in Brazil since 1937, uses the 17DD strain ${ }^{4}$. Despite the effectiveness of these vaccines, serious adverse reactions have been reported, including vaccine-associated neurotropic and viscerotropic diseases. Additionally, the YFV vaccine is contraindicated for the following risk groups: infants less than 6 months of age, women who are pregnant or lactating, and immunosuppressed individuals, e.g., individuals with thymus disease or human immunodeficiency virus (HIV) and those receiving immunosuppressive therapies ${ }^{5}$.

Because of this limitation, vaccines based on recombinant technology, such as pseudo-infectious viral particles (PIVs), have been suggested as safer alternatives ${ }^{6}$. Additionally, PIVs may be useful for serum neutralization assays, antibodydependent enhancement (ADE) evaluation, screening of antiviral compounds, and construction of viral vectors ${ }^{7-9}$.

Here, we report the development and characterization of a HEK293-YFV-prM/E-opt cell line, which constitutively 
expresses the prM/E structural proteins of YFV-17D. Additionally, the present study shows that this cell line is capable of trans-packaging the transcribed RNA of a YFV reporter replicon, thereby generating pseudo-infectious YFV reporter viral particles.

Initially, 84 nucleotides (nts) of the carboxy-terminal $\mathrm{C}$-protein gene, which functions as a signal sequence for translocation of the prM protein into the lumen of the endoplasmic reticulum ${ }^{10}$, and the complete sequences of the $\mathrm{prM} / \mathrm{E}$ genes of the YFV-17D strain were used for the constructs. Codons of the sequences were optimized for expression in eukaryotic cells (Leto 1.0 Software) and commercially synthesized (GENEART, Regensburg, Germany). The genes were removed by digestion with $N h e \mathrm{I}$ and $K p n \mathrm{I}$ and inserted into the NheI and $K p n I$ sites of the pcDNA3.1 vector, resulting in pcDNA-YFV-prM/E-opt.

To develop the HEK293-YFV-prM/E-opt cell line, $4 \times 10^{6}$ HEK293 cells were resuspended in $400 \mu \mathrm{L}$ of Cytomix (120 $\mathrm{mM} \mathrm{KCl} 2,0.15 \mathrm{mM} \mathrm{CaCl}_{2}, 10 \mathrm{mM} \mathrm{K}_{2} \mathrm{HPO}_{4}, 25 \mathrm{mM}$ HEPES, 2 $\mathrm{mM}$ EDTA, and $5 \mathrm{mM} \mathrm{MgCl}_{2} ; \mathrm{pH}$ 7.6), electroporated with 10 $\mu \mathrm{g}$ of pcDNA-YFV-prM/E-opt (4 mm cuvettes, $600 \mathrm{~V}, 100 \mu \mathrm{s}$, 4 pulses with $1 \mathrm{~s}$ interval between pulses, ECM-830, BTX), and maintained in Dulbecco's modified Eagle's medium (DMEM) with $10 \%$ fetal bovine serum (FBS), $2 \mathrm{mM}$ L-glutamine, penicillin $(100 \mathrm{U} / \mathrm{mL})$, and streptomycin $(100 \mu \mathrm{g} / \mathrm{mL})(\mathrm{GIBCO}$ BRL Life Technologies, Brazil). Two days after electroporation, geneticin (GIBCO BRL Life Technologies) $(700 \mu \mathrm{g} / \mathrm{mL})$ was added to the culture medium for selection. Selected cells were cloned into 96-well plates (one cell/well). Clone 16 best expressed the $\mathrm{prM} / \mathrm{E}$ proteins (data not shown) and was selected for characterization and trans-packaging assays. Throughout the experiments, HEK293-YFV-prM/E-opt cells were maintained in the presence of geneticin.

After obtaining the HEK293-YFV-prM/E-opt cell line, expression of the $\mathrm{prM} / \mathrm{E}$ proteins was initially analyzed by indirect immunofluorescence. The HEK293-YFV-prM/E-opt cells were seeded onto multispot slides and fixed with methanol for $5 \mathrm{~min}$ at $-20^{\circ} \mathrm{C}$. After fixation, the cells were incubated for $1 \mathrm{~h}$ at $37^{\circ} \mathrm{C}$ with mouse polyclonal antibodies, specific for flaviviruses (group B). (provided by the Instituto Evandro Chagas, Belém, Brazil), and then, washed and incubated for $1 \mathrm{~h}$ with an anti-mouse IgG secondary antibody, fluorescein isothiocyanate (FITC)conjugated secondary antibody (Sigma-Aldrich, St. Louis, MO, USA). Finally, the slides were washed and observed via fluorescence microscopy to visualize the expression of the viral structural proteins (Leica DMI 4000B) (Figure 1A).

Next, E protein expression was assessed by flow cytometry. For this, $10^{6}$ cells were placed in a polystyrene tube, washed twice with a PBS-wash solution $(0.5 \% \mathrm{BSA} / 0.1 \%$ sodium azide), and incubated for $10 \mathrm{~min}$ at $25^{\circ} \mathrm{C}$ with PBS-P solution $(0.5 \% \mathrm{BSA} / 0.1 \%$ sodium azide $/ 0.5 \%$ saponin). After incubation, cells were washed with PBS-Wash, centrifuged $(335 \times \mathrm{g}$ for 10 min), and resuspended in $20 \mu \mathrm{L}$ of an anti-YFV-17D E protein oligoclonal antibody, previously produced in our laboratory

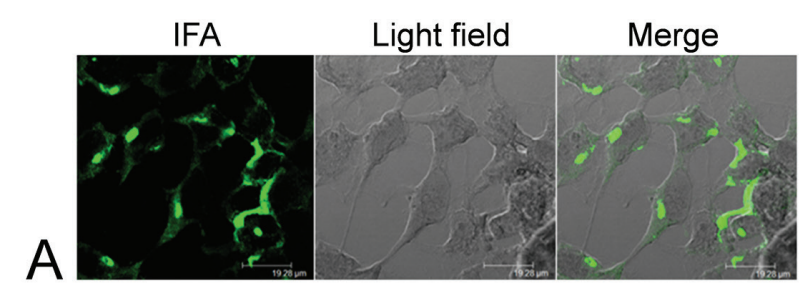

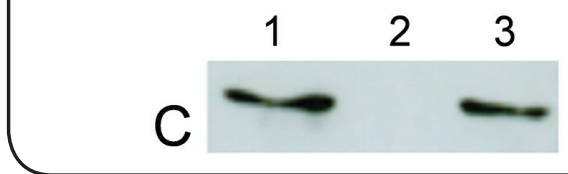

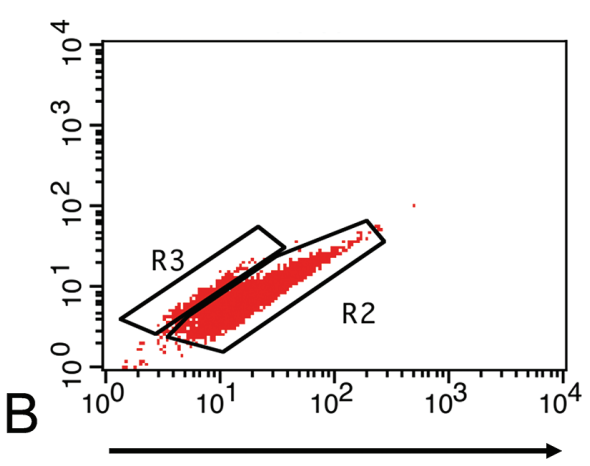

E protein expression

FIGURE 1: (A): Indirect immunofluorescence (IFA) for the analysis of prM/E protein expression by the HEK293-YFV-prM/E-opt cell line. After fixation, HEK293-YFV-prM/E-opt cells were incubated with mouse polyclonal antibodies specific for flaviviruses (group B) and then, with the anti-mouse IgG secondary antibody (FITC). Magnification, 630x.

(B): Evaluation of E protein expression by flow cytometry. HEK293-YFV-prM/E-opt cells were permeabilized and incubated with an anti-YFV-17D E protein oligoclonal antibody. Cells were then washed and incubated with an anti-lgG secondary antibody. Next, cells were washed and resuspended in formaldehyde, and the read was performed. R3: negative cells; R2: 90\% cells, positive for E protein expression.

(C): Western blotting to confirm E protein expression in the HEK293-YFV-prM/E-opt cells. The SSP and the HEK293-YFV-prM/E-opt cell pellets were lysed and subjected to SDS-PAGE. Protein bands were transferred to nitrocellulose membranes that were incubated with a YFV anti-E antibody and then with a peroxidase-conjugated antibody. The $\mathrm{E}$ protein bands were visualized with the Immobilon Western Chemiluminescent HRP substrate. 1 - supernatant from HEK293 cells infected with YFV-17D; 2 - supernatant from non-infected HEK293 cells; 3 - supernatant from HEK293-YFV-prM/E-opt cells. Western blot of the HEK293-YFV-prM/E-opt cell extract showed the same band pattern (data not shown). 
(unpublished data). After 30 min of incubation at $37^{\circ} \mathrm{C}$, the cells were washed, and the pellet resuspended in $20 \mu \mathrm{L}$ of a rabbit anti-IgG secondary antibody suspension (Sigma-Aldrich). After a second incubation of $30 \mathrm{~min}$ at $37^{\circ} \mathrm{C}$, the cells were washed and resuspended in $500 \mu \mathrm{L}$ of formaldehyde. Non-transfected cells were the negative control. Cells were read on a FACSCalibur flow cytometer (Becton Dickson Immunocytometry Systems, TX, USA) and analyzed using CellQuestPro software. The YFV E protein was expressed in $90 \%$ of the cells (Figure 1B).

The production of E protein in HEK293-YFV-prM/E-opt cells and its secretion as subviral particles (SSPs) were evaluated by western blotting. For SSPs, the cell supernatant was clarified $(600 \times \mathrm{g}$ for $10 \mathrm{~min}$ ), and the SSP pellet was obtained by ultracentrifugation at 63,000 $\times \mathrm{g}$ for $2 \mathrm{~h}$ in an Optma ${ }^{\mathrm{TM}} \mathrm{LE}-80 \mathrm{~K}$ (Beckman Coulter). The pellet was resuspended at 1:100 of the initial volume in a 5\% BSA solution in PBS. HEK293-YFV-prM/E-opt cells and the SSP pellets were lysed with Laemmli buffer (Sigma-Aldrich) and subjected to $10 \%$ SDS-PAGE. The proteins were transferred to nitrocellulose membranes, blocked with PBS/0.1\% Tween-20 with $5 \%$ nonfat dry milk, and subsequently incubated with an anti-YFV E protein oligoclonal antibody for $1 \mathrm{~h}$. Membranes were washed three times with PBS/0.1\% Tween-20 for $10 \mathrm{~min}$ and incubated with an anti-rabbit IgG peroxidase-conjugated antibody (Sigma-Aldrich) for $1 \mathrm{~h}$. The membrane was washed three times, and the E protein was detected with the Immobilon Western Chemiluminescent Horseradish Peroxidase (HRP) kit (Millipore, Billerica, MA, USA). Expression and secretion of the protein in the cell supernatant was confirmed (Figure 1C).

Finally, the trans-packaging ability of the HEK293-YFV$\mathrm{prM} / \mathrm{E}-\mathrm{opt}$ cell line was evaluated using the YFV bicistronic replicon repYFV-17D-LucIRES. Homologous recombination in Saccharomyces cerevisiae (RFY 206) ${ }^{11}$ was used to construct the repYFV-17D-LucIRES replicon by cloning four gene fragments with homologous ends into the pBSC-YFV-17D vector, digested with RsrII and NarI. In the first cistron, repYFV-17D-LucIRES has the sequence related to the $\mathrm{C}$ protein and to the coding of six amino acids of the prM protein, and the luciferase gene (Luc); in the second cistron, under the control of the internal ribosome entry site (IRES) from the encephalomyocarditis virus, the replicon has $72 \mathrm{nt}$ of the E protein signal sequence and the genes of the YFV non-structural proteins (Figure 2). This strategy enables the packaging of this replicon via the in-trans-supply of the YFV prM/E proteins when transfected into the HEK293YFV-prM/E-opt cell line. Unlike the repYFV-17D-LucIRES replicon, the monocistronic replicon repYFV-17D-Luc (Figure 2) was also constructed by homologous recombination in yeast, but did not contain the complete $\mathrm{C}$ gene region, and was used as a negative control.

HEK293-YFV-prM/E-opt cells were electroporated with $20 \mu \mathrm{g}$ of transcribed RNA from the rep-YFV17D-LucIRES or rep-YFV17D-Luc replicon. After electroporation, the cells were resuspended in DMEM and plated into six-well plates. Three days after electroporation, the supernatant from both transfections was inoculated into HEK293 and HEK293-YFV$\mathrm{prM} / \mathrm{E}-\mathrm{opt}$ cells. Replicon trans-packaging and formation of reporter PIVs were confirmed by assessing luciferase activity in the inoculated cells 48 -h post-inoculation, using a Luciferase 1000 Assay System kit on a Mithras LB 940 (Berthold Technologies). Luciferase activity of the HEK293-YFV-prM/Eopt and HEK293 cells, inoculated with the supernatant from the transfection with rep-YFV17D-LucIRES, was 55 and 3.5 times higher, respectively, than the negative control (Figure 3).

PIVs have been used for different purposes with various flaviviruses $^{6}$. PIVs from the West Nile virus (WNV) ${ }^{9}$, tickborne encephalitis virus (TBEV) ${ }^{7}$, and dengue virus(DENV $)^{8,12}$ have been developed for serum neutralization assays, vaccine formulations, and antiviral drug screening. Regarding YFV manipulations, PIVs have been recovered and used mainly for construction of chimeric PIVs for vaccination and molecular studies of viral packaging ${ }^{13,14}$.

In cell culture systems, such as in the present report, PIVs can be obtained by recombinant cell lines expressing structural viral proteins that when transfected by replicons pack the defective genomes, or also by trans-delivery of the viral proteins by transfection of replicons containing only the complementary

A

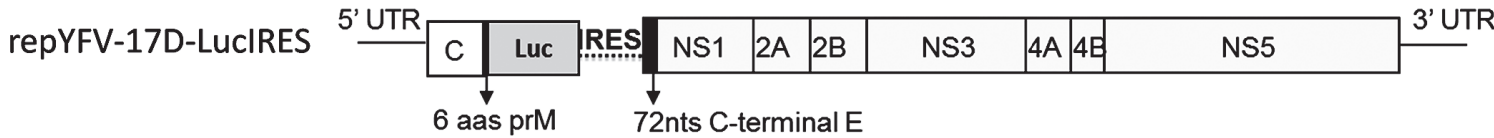

B

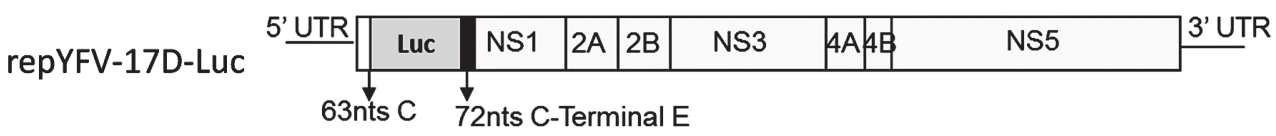

FIGURE 2: Schematic representation of the repYFV-17D-LucIRES and repYFV-17D-Luc replicons. The repYFV-17D-LucIRES (A): and repYFV-17D-Luc. (B): replicons contain all of the coding regions for the YFV non-structural proteins and are distinguished by the presence of the complete capsid region only in the repYFV-17D-LucIRES construct. YFV: yellow fever virus; Luc: luceferase gene; IRES: internal ribosome entry site; UTR: untranslated regions; NS: non-structural proteins. 


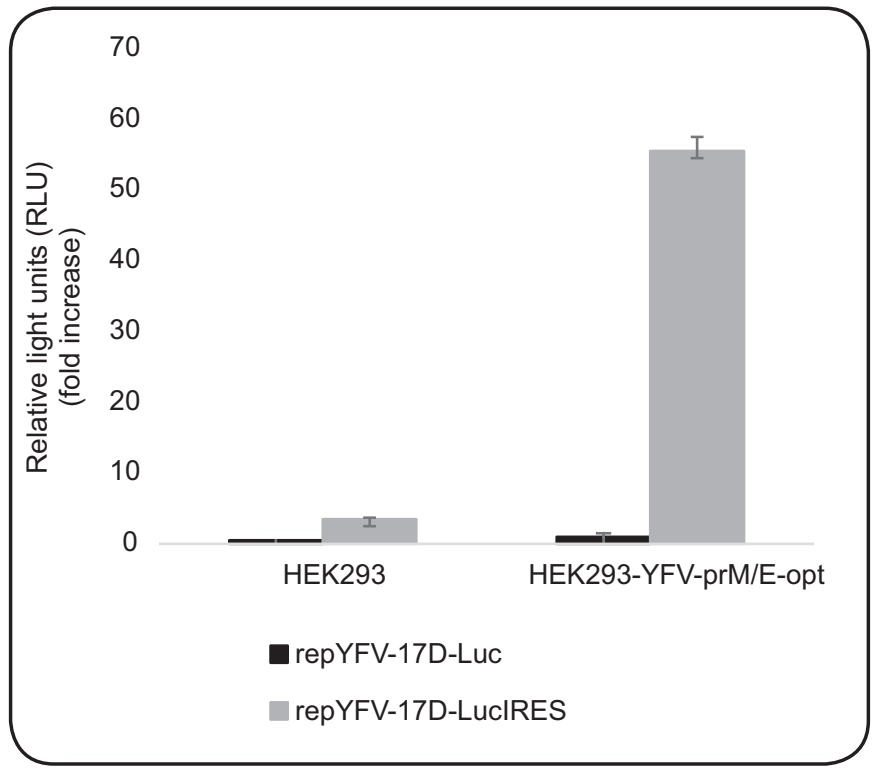

FIGURE 3: Evaluation of the trans-packaging capacity of the HEK293YFV-prM/E-opt cell line. After transfection of the HEK293-YFV-prM/E-opt cell line with the repYFV-17D-LucIRES or repYFV-17D-Luc replicon, the supernatants from both transfections were inoculated into another HEK293YFV-prM/E-opt or HEK293 cell culture. Luciferase activity was detected only in the cells infected with the supernatant from the transfection with repYFV-17D-LucIRES. YFV: yellow fever virus; Luc: luceferase gene; IRGS: internal ribosome entry site.

gene sequences necessary for viral packaging ${ }^{13-15}$. This latter strategy has been proposed both for the generation of flavivirus PIVs for vaccination purposes ${ }^{14}$ and for the recovery of alphavirus PIVs for screening of antiviral compounds ${ }^{15}$.

Thus, our work aimed to develop and characterize the packaging cell line HEK293-YFV-prM/E-opt, based on our knowledge of I) the adverse effects and the restricted target audience of live-attenuated YFV vaccines, II) the possibility of using PIVs for several biotechnological applications, and III) the use of recombinant cell lines as one of the main methods to recover PIVs.

Expression of the prM/E recombinant proteins, by the developed cell line, was confirmed by immunofluorescence, flow cytometry, and western blotting assays. In addition, flow cytometry demonstrated that $90 \%$ of the cells expressed the E protein, and western blotting confirmed secretion of the $\mathrm{E}$ protein as SSPs. Therefore, beyond expressing the complete $\mathrm{prM} / \mathrm{E}$ proteins in the PIVs, the HEK293-YFV-prM/E-opt cells' ability to secrete SSPs, which are known to be capable of inducing an efficient immune response that protects animals against the challenge of replication-competent viruses ${ }^{14}$, may suggest their future use in immunization assays.

Moreover, the trans-packaging ability of the HEK293-YFV$\mathrm{prM} / \mathrm{E}$-opt cells was confirmed by transfecting that cell line with the repYFV-17D-LucIRES replicon to generate YFV reporter PIVs. The higher luciferase activity, observed in the HEK293YFV-prM/E-opt cells inoculated with the YFV PIVs, relative to the inoculum in the corresponding wild-type (HEK293) cells, indicates that more expression of $\mathrm{prM} / \mathrm{E}$ proteins results in a greater trans-packaging capacity for the HEK293-YFV-prM/Eopt cell line. Additionally, the ability of the HEK293-YFV$\mathrm{prM} / \mathrm{E}$-opt cells to carry the repYFV-17D-LucIRES replicon highlights the possible application of this cell line as a vector for the delivery and expression of heterologous genes for vaccines or therapeutic purposes.

This strategy of heterologous gene delivery systems, through the joint use of cell lines expressing structural viral proteins and replicons, has also been successfully used for TBEV. Similar to the transfection system with HEK293-YFV-prM/E-opt, transfection of a replicon, containing the non-structural proteins of TBEV and green fluorescent protein (GFP) into BHK-21 cells expressing TBEV structural proteins, resulted in reporter $\mathrm{PIVs}^{7}$.

For the possible use of YFV reporter PIVs in the discovery of antiviral drugs, Luc is presented as a highly sensitive and easy-to-read reporter gene that is frequently used for widespread screening of antiviral compounds ${ }^{15}$.

Beyond these possibilities in applied virology, $84 \mathrm{nt}$ of the carboxy-terminal C-protein gene were added to the $\mathrm{prM} / \mathrm{E}$ sequence in the HEK293-YFV-prM/E-opt cell line, resulting in the correct translation of these proteins; this permits studying the mechanism of flavivirus translation processes.

In summary, our trans-packaging strategy is a possible platform for the recovery of YFV PIVs for future use in diagnostic assays, drug screening, immunization, viral vector construction, and viral trans-packaging research. In addition, although reporter genes, such as Luc, are frequently used for the evaluation of PIVs of several flaviviruses ${ }^{7-9}$, electron microscopy should be conducted for better characterization of the generated YFV PIVs. Finally, the HEK293-YFV-prM/E-opt cell line may also serve as a model trans-packaging strategy for the recovery of PIVs from similar viruses, especially those with vaccine manipulations and molecular virology studies still in the initial stages, such as the Zika virus.

\section{Conflict of interest}

The authors declare that there is no conflict of interest.

\section{Financial Support}

Conselho Nacional de Desenvolvimento Cientifico e Tecnológico (CNPq) and Instituto Aggeu Magalhães/Fundação Oswaldo Cruz (Fiocruz, PE).

\section{REFERENCES}

1. Lindenbach BD, Murray CL, Thiel H-J, Rice CM. Flaviviridae. In: Knipe DM, Howley PM, editors. Fields Virology, $6^{\text {th }}$ edition. New York: Lippincott Williams \& Wilkins; 2013. p. 712-46.

2. Gardner CL, Ryman KD. Yellow fever: a reemerging threat. Clin Lab Med. 2010;30(1):237-60.

3. Ministério da Saúde (MS). Secretaria de Vigilância em Saúde. COES - Febre Amarela. Portal da Saúde [Internet]. Informe No 39/2017. Brasília:: MS; 2017; 6p. [Atualizada em 4 may 2017; citada 9 may 2017]. Disponível em: http://portalarquivos.saude.gov.br/images/ pdf/2017/maio/04/COES-FEBRE-AMARELA---INFORME-39--Atualizacao-em-04maio2017.pdf 
4. Pereira RC, Silva AN, Souza MC, Silva MV, Neves PP, Silva AA, et al. An inactivated yellow fever 17DD vaccine cultivated in Vero cell cultures. Vaccine. 2015;33(35):4261-8.

5. Thomas RE, Lorenzetti DL, Spragins W, Jackson D, Williamson T. The safety of yellow fever vaccine 17D or 17DD in children, pregnant women, HIV+ individuals, and older persons: systematic review. Am J Trop Med Hyg. 2012;86(2):359-72.

6. Mason PW, Shustov AV, Frolov I. Production and characterization of vaccines based on flaviviruses defective in replication. Virology. 2006;351(2):432-43.

7. Yoshii K, Hayasaka D, Goto A, Kawakami K, Kariwa H, Takashima I. Packaging the replicon RNA of the Far-Eastern subtype of tickborne encephalitis virus into single-round infectious particles: development of a heterologous gene delivery system. Vaccine. 2005;23(30):3946-56.

8. Mukherjee S, Pierson TC, Dowd KA. Pseudo-infectious reporter virus particles for measuring antibody-mediated neutralization and enhancement of dengue virus infection. In: Padmanabhan R, Vasudevan $\mathrm{S}$, editors. Dengue. Methods in Molecular Biology (Methods and Protocols). New York: Humana Press; vol 1138, 2014. p. 75-97.

9. Zhang HL, Ye HQ, Deng CL, Liu SQ, Shi PY, Qin CF, et al. Generation and characterization of West Nile pseudo-infectious reporter virus for antiviral screening. Antiviral Res. 2017;141:38-47.
10. Patkar CG, Jones CT, Chang YH, Warrier R, Kuhn RJ. Functional requirements of the yellow fever virus capsid protein. J Virol. 2007;81(12):6471-81.

11. Silva JV, Arenhart S, Santos HF, Almeida-Queiroz SR, Silva AN, Trevisol IM, et al. Efficient assembly of full-length infectious clone of Brazilian IBDV isolate by homologous recombination in yeast. Braz J Microbiol. 2014;45(4):1555-63.

12. Suzuki R, Winkelmann ER, Mason PW. Construction and characterization of a single-cycle chimeric flavivirus vaccine candidate that protects mice against lethal challenge with dengue virus type 2. J Virol. 2009;83(4):1870-80.

13. Shustov AV, Mason PW, Frolov I. Production of pseudoinfectious yellow fever virus with a two-component genome. J Virol. 2007;81(21):11737-48.

14. Shustov AV, Frolov I. Efficient, trans-complementing packaging systems for chimeric, pseudoinfectious dengue 2/yellow fever viruses. Virology. 2010;400(1):8-17.

15. Delekta PC, Raveh A, Larsen MJ, Schultz PJ, Tamayo-Castillo G, Sherman DH, et al. The combined use of alphavirus replicons and pseudoinfectious particles for the discovery of antivirals derived from natural products. J Biomol Screen. 2015;20(5):673-80. 